\title{
Association of genetic variants with chronic kidney disease in Japanese individuals with or without hypertension or diabetes mellitus
}

\author{
TETSURO YOSHIDA ${ }^{1}$, KIMIHIKO KATO ${ }^{2}$, KIYOSHI YOKOI ${ }^{2}$, MITSUTOSHI OGURI $^{3}$, \\ SACHIRO WATANABE ${ }^{4}$, NORIFUMI METOKI ${ }^{5}$, HIDEMI YOSHIDA $^{6}$, KEI SATOH $^{6}$, \\ YUKITOSHI AOYAGI $^{7}$, YOSHINORI NOZAWA ${ }^{8}$ and YOSHIJI YAMADA $^{9}$
}

\begin{abstract}
${ }^{1}$ Department of Cardiovascular Medicine, Inabe General Hospital, Inabe; ${ }^{2}$ Department of Cardiovascular Medicine, Gifu Prefectural Tajimi Hospital, Tajimi; ${ }^{3}$ Department of Cardiology, Japanese Red Cross Nagoya First Hospital, Nagoya; ${ }^{4}$ Department of Cardiology, Gifu Prefectural General Medical Center, Gifu; ${ }^{5}$ Department of Internal Medicine, Hirosaki Stroke Center, Hirosaki; ${ }^{6}$ Department of Vascular Biology, Institute of Brain Science, Hirosaki University Graduate School of Medicine, Hirosaki; ${ }^{7}$ Department of Genomics for Longevity and Health, Tokyo Metropolitan Institute of Gerontology, Tokyo; ${ }^{8}$ Gifu International Institute of Biotechnology and Tokai Gakuin University, Kakamigahara;

${ }^{9}$ Department of Human Functional Genomics, Life Science Research Center, Mie University, Mie, Japan
\end{abstract}

Received July 21, 2009; Accepted September 8, 2009

DOI: 10.3892/etm_00000023

\begin{abstract}
Hypertension and diabetes mellitus are important risk factors for chronic kidney disease (CKD). The purpose of the present study was to identify genetic variants that confer susceptibility to CKD in individuals with or without hypertension or diabetes mellitus, thereby contributing to the personalized prevention of CKD in such individuals separately. The study population comprised 5835 unrelated Japanese individuals, including 1763 subjects with CKD and 4072 controls. The 150 polymorphisms were selected by genome-wide association studies of ischemic stroke and myocardial infarction with the use of the GeneChip Human Mapping 500K Array Set (Affymetrix). The genotypes for these polymorphisms were determined by a method that combines polymerase chain reaction and sequence-specific oligonucleotide probes with suspension array technology. The $\chi^{2}$ test, multivariable logistic regression analysis with adjustment for covariates, as well as a stepwise forward selection procedure revealed that two different polymorphisms were significantly $(\mathrm{P}<0.005)$ associated with the prevalence of CKD in individuals with or without hypertension or diabetes mellitus: the $\mathrm{A} \rightarrow \mathrm{G}$ (Lys625Arg) polymorphism of $\mathrm{CDH} 4$ (rs6142884) in individuals without diabetes mellitus, and the $\mathrm{C} \rightarrow \mathrm{T}$ polymorphism of PTPRN2 (rs1638021) in individuals with hypertension and diabetes mellitus. No polymorphism
\end{abstract}

Correspondence to: Dr Yoshiji Yamada, Department of Human Functional Genomics, Life Science Research Center, Mie University, 1577 Kurima-machiya, Tsu, Mie 514-8507, Japan

E-mail: yamada@gene.mie-u.ac.jp

Key words: genetics, polymorphism, chronic kidney disease, diabetes mellitus, hypertension was significantly associated with CKD in individuals with or without hypertension, in those with diabetes mellitus, or in those without hypertension or diabetes mellitus. Stratification of subjects based on hypertension or diabetes mellitus may thus be fundamental to achieving the personalized prevention of CKD with the use of genetic information.

\section{Introduction}

It is well known that chronic kidney disease (CKD) and endstage renal disease (ESRD), which accelerate cardiovascular disease, are associated with high mortality (1). Recent studies suggest that the risk of death is increased in individuals who have impaired renal function but do not require dialysis, compared to those who have preserved renal function $(2,3)$. Disease prevention is an important strategy for reducing the overall burden of CKD and ESRD, and the identification of markers for CKD risk is essential both for risk prediction and for potential intervention to reduce the chance of future cardiovascular events related to CKD (4).

Although genetic linkage analyses (5) and association studies (6-8) have implicated several loci and candidate genes in the predisposition to $\mathrm{CKD}$, the genes that confer susceptibility to this condition remain to be identified definitively. In addition, given ethnic differences in lifestyle and environmental factors as well as in genetic background and renal function, it is necessary to examine genetic variants related to CKD in each ethnic group. We previously showed that genetic variants that confer susceptibility to CKD differ between individuals with or without metabolic syndrome (9), with or without type 2 diabetes mellitus (10), with or without hypertension (11), or with different lipid profiles (12). To further examine whether the association of polymorphisms with CKD is influenced by the absence or presence of hypertension or diabetes mellitus, we performed an association study for 150 polymorphisms of 
144 candidate genes and CKD in 5835 Japanese individuals with or without hypertension or diabetes mellitus. The purpose of the present study was to identify genetic variants that confer susceptibility to CKD in individuals with or without hypertension or diabetes mellitus independently, and thereby to assess the genetic risk of CKD in such individuals separately.

\section{Materials and methods}

Study population. The study population comprised 5835 unrelated Japanese individuals (3309 men, 2526 women) who either visited outpatient clinics or were admitted to one of the participating hospitals (Gifu Prefectural General Medical Center and Gifu Prefectural Tajimi Hospital in Gifu Prefecture, and Hirosaki University Hospital, Reimeikyo Rehabilitation Hospital and Hirosaki Stroke Center in Aomori Prefecture, Japan) between October 2002 and March 2008 due to various symptoms or for an annual health checkup, or were recruited to a population-based prospective cohort study of aging and age-related diseases in Nakanojo, Gunma Prefecture, Japan.

Estimated glomerular filtration rate (eGFR) was calculated with the use of the simplified prediction equation derived from a modified version of that described in the Modification of Diet in Renal Disease (MDRD) Study, as proposed by the Japanese Society of Nephrology (13): eGFR ( $\left.\mathrm{ml} \mathrm{min}^{-1} 1.73 \mathrm{~m}^{-2}\right)$ $=194 \times$ [age $($ years $)]-0.287 \times$ [serum creatinine $(\mathrm{mg} / \mathrm{dl})]-1.094$ x 0.739 (if female). The National Kidney Foundation's Kidney Disease Outcomes Quality Initiative guidelines recommend a diagnosis of CKD if the eGFR is $<60 \mathrm{ml} \mathrm{min}{ }^{-1} 1.73 \mathrm{~m}^{-2}$ (4). On the basis of this criterion, 1763 subjects (1076 men, 687 women) were diagnosed with CKD. The control subjects comprised 4072 individuals (2233 men, 1839 women) recruited from among community-dwelling healthy individuals or patients who visited outpatient clinics regularly for treatment of various common diseases, with an eGFR $\geq 60 \mathrm{ml} \mathrm{min}^{-1} 1.73 \mathrm{~m}^{-2}$. Subjects with CKD and controls thus either had or did not have conventional risk factors for CKD, including hypertension (systolic blood pressure of $\geq 140 \mathrm{mmHg}$, diastolic blood pressure of $\geq 90 \mathrm{mmHg}$, or taking antihypertensive medication), diabetes mellitus (fasting blood glucose of $\geq 6.93 \mathrm{mmol} / \mathrm{l}$, hemoglobin $\mathrm{A}_{\mathrm{lc}}$ of $\geq 6.5 \%$, or taking antidiabetes medication), or hypercholesterolemia (serum total cholesterol of $\geq 5.72 \mathrm{mmol} / \mathrm{l}$ or taking lipid-lowering medication). On the basis of these criteria, 3434 and 2401 subjects were diagnosed with or without hypertension, respectively, 1710 and 4125 subjects were diagnosed with or without diabetes mellitus, respectively, and 1296 and 1987 subjects were diagnosed with or without hypertension and diabetes mellitus, respectively.

The study protocol complied with the Declaration of Helsinki and was approved by the Committees on the Ethics of Human Research of Mie University Graduate School of Medicine, Hirosaki University Graduate School of Medicine, Gifu International Institute of Biotechnology, Tokyo Metropolitan Institute of Gerontology, and participating hospitals. Written informed consent was obtained from each participant.

Selection of polymorphisms. A total of 150 polymorphisms (data not shown) were selected by genome-wide association studies of ischemic stroke and myocardial infarction (P-value for allele frequency $<1.0 \times 10^{-7}$ ) with the use of the GeneChip Human Mapping 500K Array Set (Affymetrix, Santa Clara, CA, USA) 
Table II. Characteristics of subjects with chronic kidney disease and controls among individuals with or without hypertension.

\begin{tabular}{|c|c|c|c|c|c|c|}
\hline \multirow[b]{2}{*}{ Characteristic } & \multicolumn{3}{|c|}{ With hypertension } & \multicolumn{3}{|c|}{ Without hypertension } \\
\hline & CKD & Controls & P-value & CKD & Controls & P-value \\
\hline No. of subjects & 1217 & 2217 & & 546 & 1855 & \\
\hline Age (years) & $70.9 \pm 8.9$ & $66.4 \pm 9.8$ & $<0.0001$ & $70.5 \pm 9.4$ & $63.8 \pm 11.2$ & $<0.0001$ \\
\hline Gender (male/female, \%) & $62.2 / 37.8$ & $59.7 / 40.3$ & 0.1472 & $58.4 / 41.6$ & $49.1 / 50.9$ & 0.0001 \\
\hline Body mass index $\left(\mathrm{kg} / \mathrm{m}^{2}\right)$ & $23.5 \pm 3.4$ & $23.8 \pm 3.4$ & 0.0100 & $23.3 \pm 3.3$ & $23.0 \pm 3.2$ & 0.1470 \\
\hline Current or former smoker $(\%)$ & 18.5 & 24.1 & 0.0002 & 22.2 & 24.2 & 0.3365 \\
\hline Systolic blood pressure (mmHg) & $152 \pm 27$ & $149 \pm 23$ & 0.0079 & $128 \pm 17$ & $127 \pm 16$ & 0.6248 \\
\hline Diastolic blood pressure (mmHg) & $79 \pm 16$ & $81 \pm 14$ & 0.0001 & $73 \pm 12$ & $74 \pm 11$ & 0.0267 \\
\hline Hypercholesterolemia (\%) & 31.9 & 30.5 & 0.4151 & 28.9 & 27.2 & 0.4310 \\
\hline Serum total cholesterol (mmol/l) & $5.21 \pm 1.04$ & $5.18 \pm 1.02$ & 0.2958 & $5.21 \pm 1.00$ & $5.16 \pm 0.95$ & 0.2803 \\
\hline Serum triglyceride $(\mathrm{mmol} / \mathrm{l})$ & $1.74 \pm 1.08$ & $1.69 \pm 1.14$ & 0.0062 & $1.62 \pm 0.98$ & $1.48 \pm 1.02$ & $<0.0001$ \\
\hline Serum HDL-cholesterol (mmol/l) & $1.29 \pm 0.40$ & $1.34 \pm 0.38$ & $<0.0001$ & $1.39 \pm 0.39$ & $1.46 \pm 0.40$ & 0.0002 \\
\hline Serum LDL-cholesterol (mmol/l) & $3.13 \pm 0.95$ & $3.05 \pm 0.92$ & 0.0110 & $3.06 \pm 0.88$ & $3.00 \pm 0.81$ & 0.1952 \\
\hline Diabetes mellitus (\%) & 42.6 & 35.1 & $<0.0001$ & 21.4 & 16.0 & 0.0032 \\
\hline Fasting plasma glucose $(\mathrm{mmol} / \mathrm{l})$ & $7.23 \pm 3.27$ & $7.14 \pm 3.19$ & 0.4658 & $6.50 \pm 2.96$ & $6.29 \pm 2.52$ & 0.1446 \\
\hline Blood glycosylated hemoglobin (\%) & $6.13 \pm 1.62$ & $6.06 \pm 1.60$ & 0.0374 & $5.73 \pm 1.50$ & $5.62 \pm 1.38$ & 0.2032 \\
\hline Serum creatinine $(\mu \mathrm{mol} / \mathrm{l})$ & $120.7 \pm 137.9$ & $62.3 \pm 12.8$ & $<0.0001$ & $91.9 \pm 26.5$ & $61.0 \pm 12.3$ & $<0.0001$ \\
\hline eGFR (ml min-1 $\left.1.73 \mathrm{~m}^{-2}\right)$ & $47.3 \pm 11.8$ & $79.2 \pm 16.2$ & $<0.0001$ & $50.9 \pm 8.10$ & $79.4 \pm 17.8$ & $<0.0001$ \\
\hline
\end{tabular}

Quantitative data are the means \pm SD. CKD, chronic kidney disease; LDL, low density lipoprotein; HDL, high density lipoprotein; eGFR, estimated glomerular filtration rate.

(14). The relationship of these polymorphisms to CKD was not previously examined in our studies $(9-12,15,16)$.

Genotyping of polymorphisms. Venous blood $(7 \mathrm{ml})$ was collected into tubes containing $50 \mathrm{mmol} / \mathrm{l}$ ethylenediaminetetraacetic acid (disodium salt), and genomic DNA was isolated with a kit (Genomix; Talent, Trieste, Italy). Genotypes of the 150 polymorphisms were determined at G\&G Science (Fukushima, Japan) by a method that combines polymerase chain reaction and sequence-specific oligonucleotide probes with suspension array technology (Luminex, Austin, TX, USA). Primers, probes and other conditions for the genotyping of polymorphisms significantly associated with CKD are shown in Table I. Detailed genotyping methodology was as described previously (17).

Statistical analysis. Quantitative data were compared between subjects with CKD and controls by the unpaired Student's t-test. Categorical data were compared by the $\chi^{2}$ test. Allele frequencies were estimated by the gene counting method, and the $\chi^{2}$ test was used to identify departures from Hardy-Weinberg equilibrium. In the initial screen, the allele frequencies of each polymorphism were compared between subjects with CKD and controls by the $\chi^{2}$ test. Polymorphisms with a P-value for allele frequency of $<0.005$ were further examined by multivariable logistic regression analysis with adjustment for covariates. Multivariable logistic regression analysis was thus performed with CKD as a dependent variable, and independent variables including age, gender ( 0 , woman; 1 , man), body mass index (BMI), smoking status (0, non-smoker; 1 , smoker), history of hypertension, diabetes mellitus or hypercholesterolemia
( 0 , no history; 1 , positive history), and the genotype of each polymorphism. Subsequenty, the P-value, odds ratio and 95\% confidence interval were calculated. Each genotype was assessed according to dominant, recessive and additive genetic models. Additive models included the additive 1 (heterozygotes vs. wild-type homozygotes) and additive 2 (variant homozygotes vs. wild-type homozygotes) models, which were analyzed simultaneously using a single statistical model. We also performed a stepwise forward selection procedure to examine the effects of the genotypes as well as of other covariates on CKD. Each genotype was examined according to a dominant or recessive model on the basis of statistical significance in the multivariable logistic regression analysis. The P-levels for inclusion in and exclusion from the model were 0.25 and 0.1 , respectively. Given the multiple comparisons of genotypes with CKD, we adopted the criterion of a P-value of $<0.005$ for statistical significance of association. For other clinical background data, a P-value of $<0.05$ was considered statistically significant. Statistical significance was examined by two-sided tests performed with JMP version 6.0 and JMP Genomics version 3.2 software (SAS Institute, Cary, NC, USA).

\section{Results}

Genetic variants related to CKD in individuals with or without hypertension. The characteristics of the subjects with or without hypertension are shown in Table II. For individuals with hypertension, age, systolic blood pressure, serum concentrations of triglycerides and low density lipoprotein 
Table III. Characteristics of subjects with chronic kidney disease and controls among individuals with or without diabetes mellitus.

\begin{tabular}{|c|c|c|c|c|c|c|}
\hline \multirow[b]{2}{*}{ Characteristic } & \multicolumn{3}{|c|}{ With diabetes mellitus } & \multicolumn{3}{|c|}{ Without diabetes mellitus } \\
\hline & CKD & Controls & P-value & CKD & Controls & P-value \\
\hline No. of subjects & 635 & 1075 & & 1128 & 2997 & \\
\hline Age (years) & $70.2 \pm 9.1$ & $65.5 \pm 9.9$ & $<0.0001$ & $71.1 \pm 9.0$ & $65.1 \pm 10.7$ & $<0.0001$ \\
\hline Gender (male/female, \%) & $66.1 / 33.9$ & $66.0 / 34.0$ & 0.9679 & $58.2 / 41.8$ & $50.8 / 49.2$ & $<0.0001$ \\
\hline Body mass index $\left(\mathrm{kg} / \mathrm{m}^{2}\right)$ & $23.7 \pm 3.6$ & $23.9 \pm 3.6$ & 0.1981 & $23.3 \pm 3.3$ & $23.3 \pm 3.2$ & 0.9413 \\
\hline Current or former smoker $(\%)$ & 19.4 & 25.3 & 0.0049 & 19.8 & 23.7 & 0.0073 \\
\hline Hypertension (\%) & 81.6 & 72.4 & $<0.0001$ & 62.0 & 48.0 & $<0.0001$ \\
\hline Systolic blood pressure (mmHg) & $150 \pm 28$ & $143 \pm 24$ & $<0.0001$ & $143 \pm 26$ & $139 \pm 23$ & $<0.0001$ \\
\hline Diastolic blood pressure (mmHg) & $78 \pm 15$ & $78 \pm 14$ & 0.8850 & $78 \pm 15$ & $79 \pm 13$ & 0.0044 \\
\hline Hypercholesterolemia (\%) & 32.0 & 32.0 & 0.9892 & 30.4 & 28.0 & 0.1213 \\
\hline Serum total cholesterol (mmol/l) & $5.22 \pm 1.11$ & $5.21 \pm 1.13$ & 0.4798 & $5.20 \pm 0.97$ & $5.15 \pm 0.94$ & 0.1803 \\
\hline Serum triglyceride $(\mathrm{mmol} / \mathrm{l})$ & $1.86 \pm 1.25$ & $1.80 \pm 1.26$ & 0.0681 & $1.62 \pm 0.92$ & $1.52 \pm 1.01$ & $<0.0001$ \\
\hline Serum HDL-cholesterol (mmol/l) & $1.25 \pm 0.41$ & $1.27 \pm 0.35$ & 0.0315 & $1.36 \pm 0.39$ & $1.44 \pm 0.40$ & $<0.0001$ \\
\hline Serum LDL-cholesterol (mmol/l) & $3.14 \pm 1.00$ & $3.10 \pm 1.00$ & 0.2081 & $3.09 \pm 0.88$ & $3.00 \pm 0.82$ & 0.0085 \\
\hline Fasting plasma glucose (mmol/l) & $9.39 \pm 4.05$ & $9.57 \pm 4.03$ & 0.2702 & $5.60 \pm 1.14$ & $5.70 \pm 1.31$ & 0.3255 \\
\hline Blood glycosylated hemoglobin (\%) & $7.10 \pm 1.95$ & $7.27 \pm 1.99$ & 0.1052 & $5.22 \pm 0.39$ & $5.21 \pm 0.48$ & 0.1372 \\
\hline Serum creatinine $(\mu \mathrm{mol} / \mathrm{l})$ & $119.7 \pm 114.5$ & $62.3 \pm 13.0$ & $<0.0001$ & $107.3 \pm 117.1$ & $61.5 \pm 12.4$ & $<0.0001$ \\
\hline eGFR $\left(\mathrm{ml} \mathrm{min} \mathrm{min}^{-1} 1.73 \mathrm{~m}^{-2}\right)$ & $46.5 \pm 11.8$ & $81.2 \pm 17.0$ & $<0.0001$ & $49.5 \pm 10.3$ & $78.6 \pm 16.9$ & $<0.0001$ \\
\hline
\end{tabular}

Quantitative data are the means \pm SD. CKD, chronic kidney disease; LDL, low density lipoprotein; HDL, high density lipoprotein; eGFR, estimated glomerular filtration rate.

Table IV. Genotype distributions of SNPs significantly associated with chronic kidney disease among individuals with or without diabetes mellitus as determined by the $\chi^{2}$ test.

\begin{tabular}{|c|c|c|c|c|c|}
\hline Gene symbol & SNP & dbSNP & CKD $(\%)$ & Controls $(\%)$ & P-value \\
\hline \multicolumn{6}{|c|}{ With diabetes mellitus } \\
\hline \multirow[t]{4}{*}{$P L A 2 G 3$} & $\mathrm{~A} \rightarrow \mathrm{G}$ & rs5753472 & & & 0.0011 \\
\hline & AA & & $174(27.5)$ & $234(22.0)$ & \\
\hline & $\mathrm{AG}$ & & $323(51.0)$ & $535(50.4)$ & \\
\hline & GG & & $136(21.5)$ & $293(27.6)$ & \\
\hline \multirow[t]{4}{*}{$R U V B L 2$} & $\mathrm{C} \rightarrow \mathrm{T}$ & rs1062708 & & & 0.0031 \\
\hline & $\mathrm{CC}$ & & $218(34.4)$ & $302(28.5)$ & \\
\hline & $\mathrm{CT}$ & & $303(47.9)$ & $525(49.4)$ & \\
\hline & $\mathrm{TT}$ & & $112(17.7)$ & $235(22.1)$ & \\
\hline \multicolumn{6}{|c|}{ Without diabetes mellitus } \\
\hline \multirow[t]{4}{*}{$\mathrm{CDH} 4$} & $A \rightarrow G$ & rs6142884 & & & 0.0041 \\
\hline & AA & & $19(1.7)$ & $51 \quad(1.7)$ & \\
\hline & $\mathrm{AG}$ & & $292(26.1)$ & $633(21.3)$ & \\
\hline & GG & & $808(72.2)$ & $2292(77.0)$ & \\
\hline
\end{tabular}

P-value for allele frequency $<0.005$. CKD, chronic kidney disease.

(LDL)-cholesterol, blood glycosylated hemoglobin content and the prevalence of diabetes mellitus were greater, whereas BMI, the percentage of smokers, diastolic blood pressure and serum concentration of high density lipoprotein (HDL)cholesterol were lower, in subjects with CKD than in controls.
For individuals without hypertension, age, the frequency of male subjects, serum concentration of triglycerides and the prevalence of diabetes mellitus were greater, whereas diastolic blood pressure and serum concentration of HDL-cholesterol were lower, in subjects with CKD than in controls. 
Table VI. Effects of genotypes and other characteristics on chronic kidney disease among individuals with or without diabetes mellitus determined by a stepwise forward selection procedure.

\begin{tabular}{lrc}
\hline Variable & P-value & $R^{2}$ \\
\hline With diabetes mellitus & & \\
Age & $<0.0001$ & 0.0433 \\
Hypertension & 0.0003 & 0.0059 \\
Without diabetes mellitus & & \\
Age & $<0.0001$ & 0.0603 \\
Hypertension & $<0.0001$ & 0.0079 \\
Smoking & $<0.0001$ & 0.0041 \\
Gender & 0.0002 & 0.0028 \\
CDH4 (GG vs. AA + AG) & 0.0029 & 0.0019 \\
\hline
\end{tabular}

$R^{2}$, contribution rate. $\mathrm{P}<0.005$

Comparison of allele frequencies with the $\chi^{2}$ test revealed that the $\mathrm{C} \rightarrow \mathrm{T}$ polymorphism of $F 10$ (rs5962) was significantly $(\mathrm{P}=0.0014)$ associated with $\mathrm{CKD}$ in individuals without hypertension, while no polymorphism was significantly associated with CKD in individuals with hypertension (data not shown). Multivariable logistic regression analysis with adjustment for age, gender, BMI, smoking status and the prevalence of diabetes mellitus and hypercholesterolemia revealed that no polymorphism was significantly $(\mathrm{P}<0.005)$ associated with CKD in individuals without hypertension (data not shown).

A stepwise forward selection procedure was performed to examine the effects of genotypes for the polymorphism associated with CKD by the $\chi^{2}$ test, as well as the effects of age, gender, BMI, smoking status and the prevalence of diabetes mellitus and hypercholesterolemia on CKD. For individuals without hypertension, age, BMI, gender and the F10 genotype (dominant model), in descending order of statistical significance, were significant $(\mathrm{P}<0.005)$ and independent determinants of CKD (data not shown).

Genetic variants related to $C K D$ in individuals with or without diabetes mellitus. The characteristics of the subjects with or without diabetes mellitus are shown in Table III. For individuals with diabetes mellitus, age, systolic blood pressure and the prevalence of hypertension were greater, whereas the percentage of smokers and serum concentration of HDLcholesterol were lower in subjects with CKD than in controls. For individuals without diabetes mellitus, age, the frequency of male subjects, systolic blood pressure, serum concentrations of triglycerides and LDL-cholesterol, and the prevalence of hypertension were greater, whereas the percentage of smokers, diastolic blood pressure and serum concentration of HDLcholesterol were lower in subjects with CKD than in controls.

Comparison of allele frequencies with the $\chi^{2}$ test revealed that two or one polymorphisms were significantly $(\mathrm{P}<0.005)$ associated with CKD in individuals with or without diabetes mellitus, respectively (Table IV). Multivariable logistic regression analysis with adjustment for age, gender, BMI, smoking status and the prevalence of hypertension and hypercholester- 
Table VII. Characteristics of subjects with chronic kidney disease and controls among individuals with or without hypertension and diabetes mellitus.

\begin{tabular}{|c|c|c|c|c|c|c|}
\hline \multirow[b]{2}{*}{ Characteristic } & \multicolumn{3}{|c|}{ With hypertention and diabetes mellitus } & \multicolumn{3}{|c|}{ With hypertention or diabetes mellitus } \\
\hline & CKD & Controls & P-value & CKD & Controls & P-value \\
\hline No. of subjects & 518 & 778 & & 429 & 1558 & \\
\hline Age (years) & $70.2 \pm 9.0$ & $66.1 \pm 9.6$ & $<0.0001$ & $70.6 \pm 9.4$ & $63.7 \pm 11.3$ & $<0.0001$ \\
\hline Gender (male/female, \%) & $66.0 / 34.0$ & $66.3 / 33.7$ & 0.9107 & $56.2 / 43.8$ & $46.0 / 54.0$ & 0.0002 \\
\hline Body mass index $\left(\mathrm{kg} / \mathrm{m}^{2}\right)$ & $23.7 \pm 3.5$ & $24.1 \pm 3.6$ & 0.0366 & $23.2 \pm 3.2$ & $23.0 \pm 3.1$ & 0.2602 \\
\hline Current or former smoker $(\%)$ & 19.3 & 24.7 & 0.0233 & 22.8 & 23.6 & 0.7369 \\
\hline Systolic blood pressure $(\mathrm{mmHg})$ & $155 \pm 27$ & $150 \pm 23$ & 0.0015 & $129 \pm 18$ & $128 \pm 17$ & 0.3841 \\
\hline Diastolic blood pressure (mmHg) & $80 \pm 15$ & $80 \pm 15$ & 0.4785 & $74 \pm 12$ & $75 \pm 11$ & 0.1781 \\
\hline Hypercholesterolemia (\%) & 33.2 & 32.9 & 0.9105 & 29.6 & 26.8 & 0.2430 \\
\hline Serum total cholesterol $(\mathrm{mmol} / \mathrm{l})$ & $5.25 \pm 1.12$ & $5.23 \pm 1.11$ & 0.4068 & $5.24 \pm 0.97$ & $5.16 \pm 0.90$ & 0.1413 \\
\hline Serum triglyceride $(\mathrm{mmol} / \mathrm{l})$ & $1.88 \pm 1.26$ & $1.84 \pm 1.33$ & 0.0624 & $1.59 \pm 0.91$ & $1.44 \pm 1.00$ & $<0.0001$ \\
\hline Serum HDL-cholesterol (mmol/l) & $1.25 \pm 0.42$ & $1.27 \pm 0.35$ & 0.0412 & $1.40 \pm 0.39$ & $1.49 \pm 0.40$ & 0.0007 \\
\hline Serum LDL-cholesterol (mmol/l) & $3.18 \pm 1.01$ & $3.12 \pm 1.02$ & 0.1437 & $3.08 \pm 0.85$ & $2.99 \pm 0.79$ & 0.0819 \\
\hline Fasting plasma glucose (mmol/l) & $9.28 \pm 3.91$ & $9.58 \pm 4.00$ & 0.1190 & $5.56 \pm 1.13$ & $5.66 \pm 1.38$ & 0.8941 \\
\hline Blood glycosylated hemoglobin (\%) & $6.99 \pm 1.89$ & $7.19 \pm 1.92$ & 0.1035 & $5.17 \pm 0.38$ & $5.18 \pm 0.48$ & 0.8077 \\
\hline Serum creatinine $(\mu \mathrm{mol} / \mathrm{l})$ & $124.2 \pm 125.4$ & $62.3 \pm 13.1$ & $<0.0001$ & $89.8 \pm 24.2$ & $60.8 \pm 12.2$ & $<0.0001$ \\
\hline $\mathrm{eGFR}\left(\mathrm{ml} \mathrm{min} \mathrm{mi}^{-1} 1.73 \mathrm{~m}^{-2}\right)$ & $46.0 \pm 12.1$ & $81.1 \pm 16.9$ & $<0.0001$ & $49.5 \pm 10.3$ & $78.6 \pm 16.9$ & $<0.0001$ \\
\hline
\end{tabular}

Quantitative data are the means \pm SD. CKD, chronic kidney disease; LDL, low density lipoprotein; HDL, high density lipoprotein; eGFR, estimated glomerular filtration rate.

Table VIII. Genotype distributions of SNPs significantly associated with chronic kidney disease among individuals with or without hypertension and diabetes mellitus as determined by the $\chi^{2}$ test.

\begin{tabular}{|c|c|c|c|c|c|}
\hline Gene symbol & SNP & dbSNP & CKD (\%) & Controls (\%) & P-value \\
\hline \multicolumn{6}{|c|}{$\begin{array}{l}\text { With hypertension } \\
\text { and diabetes mellitus }\end{array}$} \\
\hline \multirow[t]{4}{*}{$R U V B L 2$} & $\mathrm{C} \rightarrow \mathrm{T}$ & rs 1062708 & & & 0.0027 \\
\hline & $\mathrm{CC}$ & & $174(33.7)$ & 208 (26.9) & \\
\hline & $\mathrm{CT}$ & & $250(48.5)$ & $386(50.0)$ & \\
\hline & TT & & $92(17.8)$ & $178(23.1)$ & \\
\hline \multirow[t]{4}{*}{ ZFP30 } & $\mathrm{A} \rightarrow \mathrm{G}$ & rs 1478462 & & & 0.0033 \\
\hline & $\mathrm{AA}$ & & $421(82.1)$ & $584(75.8)$ & \\
\hline & $\mathrm{AG}$ & & 89 (17.3) & $173(22.4)$ & \\
\hline & GG & & $3(0.6)$ & 14 & \\
\hline \multirow[t]{4}{*}{ PTPRN2 } & $\mathrm{C} \rightarrow \mathrm{T}$ & rs 1638021 & & & 0.0042 \\
\hline & $\mathrm{CC}$ & & $293(56.8)$ & $371(48.1)$ & \\
\hline & $\mathrm{CT}$ & & $181(35.1)$ & $325(42.1)$ & \\
\hline & $\mathrm{TT}$ & & $42 \quad(8.1)$ & $76 \quad(9.8)$ & \\
\hline \multicolumn{6}{|c|}{$\begin{array}{l}\text { Without hypertension } \\
\text { or diabetes mellitus }\end{array}$} \\
\hline \multirow[t]{4}{*}{ JPH3 } & $\mathrm{C} \rightarrow \mathrm{G}$ & rs 2562059 & & & 0.0027 \\
\hline & $\mathrm{CC}$ & & 309 (72.7) & $1021(66.2)$ & \\
\hline & $\mathrm{CG}$ & & $112(26.4)$ & $470(30.5)$ & \\
\hline & GG & & $4 \quad(0.9)$ & $51 \quad(3.3)$ & \\
\hline
\end{tabular}

P-value for allele frequency of $<0.005$. 
olemia revealed that the $\mathrm{A} \rightarrow \mathrm{G}$ polymorphism of PLA2G3 (rs5753472, additive 2 model) and the $\mathrm{C} \rightarrow \mathrm{T}$ polymorphism of RUVBL2 (rs1062708, additive 2 model) were significantly $(\mathrm{P}<0.005)$ associated with $\mathrm{CKD}$ in individuals with diabetes mellitus, and that the $\mathrm{A} \rightarrow \mathrm{G}$ polymorphism of $\mathrm{CDH} 4$ (rs6142884, recessive model) was significantly associated with CKD in individuals without diabetes mellitus (Table V).

A stepwise forward selection procedure was performed to examine the effects of genotypes for the polymorphism associated with CKD by the $\chi^{2}$ test, as well as the effects of age, gender, BMI, smoking status and the prevalence of hypertension and hypercholesterolemia on CKD (Table VI). For individuals with diabetes mellitus, age and hypertension were a significant $(\mathrm{P}<0.005)$ and independent determinant of CKD. For individuals without diabetes mellitus, age, hypertension, smoking, gender and the $\mathrm{CDH} 4$ genotype (recessive model), in descending order of statistical significance, were significant and independent determinants of CKD.

Genetic variants related to CKD in individuals with or without hypertension and diabetes mellitus. The characteristics of the subjects with hypertension and diabetes mellitus or without these conditions are shown in Table VII. For individuals with hypertension and diabetes mellitus, age and systolic blood pressure were greater, whereas BMI, the percentage of smokers and serum concentration of HDL-cholesterol were lower in subjects with CKD than in controls. For individuals without hypertension or diabetes mellitus, age, the frequency of male subjects and serum concentration of triglycerides were greater, whereas the serum concentration of HDL-cholesterol was lower in subjects with CKD than in controls.

Comparison of allele frequencies with the $\chi^{2}$ test revealed that three or one polymorphisms were significantly $(\mathrm{P}<0.005)$ associated with CKD in individuals with hypertension and diabetes mellitus or without these conditions, respectively (Table VIII). Multivariable logistic regression analysis with adjustment for age, gender, BMI, smoking status and the prevalence of hypercholesterolemia revealed that the $\mathrm{C} \rightarrow \mathrm{T}$ polymorphism of RUVBL2 (rs1062708, additive 2 model) and the $\mathrm{C} \rightarrow \mathrm{T}$ polymorphism of PTPRN2 (rs1638021, dominant model) were significantly $(\mathrm{P}<0.005)$ associated with $\mathrm{CKD}$ in individuals with hypertension and diabetes mellitus (Table IX). No polymorphism was significantly associated with CKD in individuals without hypertension or diabetes mellitus.

A stepwise forward selection procedure was performed to examine the effects of genotypes for the polymorphism associated with CKD by the $\chi^{2}$ test, as well as the effects of age, gender, BMI, smoking status and the prevalence of hypercholesterolemia on CKD (Table X). For individuals with hypertension and diabetes mellitus, age and PTPRN2 genotype (dominant model) were significant $(\mathrm{P}<0.005)$ and independent determinants of CKD. For individuals without hypertension or diabetes mellitus, age, BMI, gender and the JPH3 genotype (recessive model), in descending order of statistical significance, were significant $(\mathrm{P}<0.005)$ and independent determinants of CKD.

Finally, we examined whether the genotype distributions for the polymorphisms associated with CKD were in HardyWeinberg equilibrium. The genotype distributions for the $\mathrm{A} \rightarrow \mathrm{G}$ polymorphism of $\mathrm{CDH} 4$ (subjects with $\mathrm{CKD}, \mathrm{P}=0.2050$; controls, $\mathrm{P}=0.3414)$ and the $\mathrm{C} \rightarrow \mathrm{T}$ polymorphism of PTPRN2 (subjects with $\mathrm{CKD}, \mathrm{P}=0.0658$; controls, $\mathrm{P}=0.6959$ ) were in 
Table X. Effects of genotypes and other characteristics on chronic kidney disease among individuals with or without hypertension and diabetes mellitus determined by a stepwise forward selection procedure.

\begin{tabular}{lcc}
\hline Variable & P-value & $R^{2}$ \\
\hline $\begin{array}{l}\text { With hypertension } \\
\text { and diabetes mellitus }\end{array}$ & & \\
Age & $<0.0001$ & 0.0368 \\
PTPRN2 (CT + TT vs. CC) & 0.0044 & 0.0048 \\
Without hypertension & & \\
or diabetes mellitus & & \\
Age & $<0.0001$ & 0.0676 \\
Body mass index & 0.0005 & 0.0059 \\
Gender & 0.0034 & 0.0042 \\
JPH3 (GG vs. CC + CG) & 0.0036 & 0.0042 \\
\hline
\end{tabular}

$R^{2}$, contribution rate. $\mathrm{P}<0.005$

Hardy-Weinberg equilibrium both in subjects with CKD and in controls.

\section{Discussion}

We examined the possible relationship between 150 polymorphisms of 144 genes with the prevalence of CKD in individuals with or without hypertension or diabetes mellitus. Our association study with three steps of analysis $\left(\chi^{2}\right.$ test, multivariable logistic regression analysis and stepwise forward selection procedure) revealed that two polymorphisms were significantly associated with CKD: the $\mathrm{A} \rightarrow \mathrm{G}$ (Lys625Arg) polymorphism of $\mathrm{CDH} 4$ (rs6142884) in individuals without diabetes mellitus, and the $\mathrm{C} \rightarrow \mathrm{T}$ polymorphism of PTPRN2 (rs1638021) in individuals with hypertension and diabetes mellitus.

The cadherin 4 , type 1 , R-cadherin $(\mathrm{CDH} 4)$ gene is a member of a family of cell surface glycoproteins that mediate calcium-dependent cell-cell adhesion and are considered to play an important role in a wide range of cell-cell interactions (18). Previous reports suggest that $\mathrm{CDH} 4$ may act as a tumor suppressor gene in human gastrointestinal tumors and may potentially be used as a marker for the early diagnosis of gastrointestinal tumorigenesis (19). In addition, $\mathrm{CDH} 4$ has been shown in animal studies to play an important role in neural tract and synaptic development $(20,21)$. In the present study, we demonstrated that the $\mathrm{A} \rightarrow \mathrm{G}$ (Lys625Arg) polymorphism of $\mathrm{CDH} 4$ (rs6142884) was significantly associated with CKD in individuals without diabetes mellitus, with the $G$ allele protecting against this condition. The Lys625Arg polymorphism is located in the cadherin repeat domain, which exists as repeats in extracellular regions thought to mediate cell-cell contact when bound to calcium. The association of the $A \rightarrow G$ (Lys625Arg) polymorphism with CKD might be attributable to effects on cellular adhesion, though the mechanism responsible for this association remains to be elucidated.

The protein tyrosine phosphatase, receptor type, $\mathrm{N}$ polypeptide 2 (PTPRN2) gene encodes a 1015-amino acid polypeptide with a single transmembrane and one putative tyrosine phosphatase catalytic domain (22). PTPRN2, which was cloned from a rat insulinoma cDNA library (22), is $74 \%$ identical to the ICA512/IA-2 autoantigen of type 1 diabetes mellitus in the cytoplasmic domain, but only $29 \%$ identical in the luminal domain (23). Previous reports suggest that 48 and $61 \%$ of sera from patients with new onset type 1 diabetes mellitus are positive for autoantibodies to the full-length and cytoplasmic domain of PTPRN2, respectively (23). Therefore, PTPRN2 has been considered a major autoantigen for type 1 diabetes mellitus, and is thus believed to be involved in the pathogenesis of this condition (23). We have now shown that the $\mathrm{C} \rightarrow \mathrm{T}$ polymorphism of PTPRN2 (rs1638021) was significantly associated with CKD in individuals with hypertension and diabetes mellitus, with the $\mathrm{T}$ allele protecting against this condition, though the underlying mechanism remains unclear.

Our study had several limitations: (i) we used eGFR instead of directly measured GFR to define CKD. (ii) We did not obtain information on the underlying renal disease in each subject with CKD, though such information could be obtained by detailed clinical examination, including renal biopsy; however, these diagnostic procedures are not considered feasible in a study with subjects recruited from the general population. (iii) It is possible that one or more of the polymorphisms associated with CKD in the present study are in linkage disequilibrium with other polymorphisms in the same gene or in other nearby genes that are actually responsible for the development of this condition. (iv) The functional relevance of the identified polymorphisms to gene transcription or to protein structure or function was not determined in the present study. (v) Although we adopted the criterion of $\mathrm{P}<0.005$ for association to compensate for the multiple comparisons of genotypes with CKD, it is not possible to completely exclude potential statistical errors such as false positives. (vi) Although a previous study showed smoking to be a risk factor for CKD (24), the frequency of smoking was lower in subjects with CKD than in controls in the present study. Selection bias thus could not be completely excluded in the present study.

In conclusion, our results suggest that genetic variants that confer susceptibility to CKD differ among individuals with or without hypertension or diabetes mellitus. Stratification of subjects based on hypertension or diabetes mellitus may thus be fundamental to achieving the personalized prevention of CKD with the use of genetic information. Given that our present study may be considered hypothesis generating, validation of our findings will require their replication with independent subject panels.

\section{Acknowledgements}

This work was supported in part by Grants-in-Aid for Scientific Research from the Ministry of Education, Culture, Sports, Science and Technology of Japan (nos. 18209023, 18018021 and 19659149 to Y.Y.).

\section{References}

1. Foley RN, Parfrey PS and Sarnak MJ: Clinical epidemiology of cardiovascular disease in chronic renal disease. Am J Kidney Dis 32: 112-119, 1998

2. Go AS, Chertow GM, Fan D, McCulloch CE and Hsu CY: Chronic kidney disease and the risks of death, cardiovascular events and hospitalization. N Engl J Med 351: 1296-1305, 2004. 
3. Ruilope LM, Salvetti A, Jamerson K, et al: Renal function and intensive lowering of blood pressure in hypertensive participants of the hypertension optimal treatment (HOT) study. J Am Soc Nephrol 12: 218-225, 2001.

4. National Kidney Foundation: K/DOQI clinical practice guidelines for chronic kidney disease: evaluation, classification, and stratification. Am J Kidney Dis 39: 1-266, 2002.

5. Gharavi AG, Yan Y, Scolari F, et al: IgA nephropathy, the most common cause of glomerulonephritis, is linked to 6q22-23. Nat Genet 26: 354-357, 2000.

6. Hanson RL, Craig DW and Millis MP: Identification of PVT1 as a candidate gene for end-stage renal disease in type 2 diabetes using a pooling-based genome-wide single nucleotide polymorphism association study. Diabetes 56: 975-983, 2007.

7. Doi K, Noiri E, Nakao A, Fujita T, Kobayashi S and Tokunaga K: Functional polymorphisms in the vascular endothelial growth factor gene are associated with development of end-stage renal disease in males. J Am Soc Nephrol 17: 823-830, 2006.

8. Wetmore JB, Hung AM, Lovett DH, Sen S, Quershy O and Johansen KL: Interleukin-1 gene cluster polymorphisms predict risk of ESRD. Kidney Int 68: 278-284, 2005.

9. Yoshida T, Kato K, Fujimaki T, et al: Association of a polymorphism of the apolipoprotein $\mathrm{E}$ gene with chronic kidney disease in Japanese individuals with metabolic syndrome. Genomics 93 : 221-226, 2009.

10. Yoshida T, Kato K, Yokoi K, et al: Association of genetic variants with chronic kidney disease in Japanese individuals with type 2 diabetes mellitus. Int J Mol Med 23: 529-537, 2009.

11. Yoshida T, Kato K, Yokoi K, et al: Association of candidate gene polymorphisms with chronic kidney disease in Japanese individuals with hypertension. Hypertens Res 32: 411-418, 2009.

12. Yoshida T, Kato K, Yokoi K, et al: Association of genetic variants with chronic kidney disease in individuals with different lipid profiles. Int J Mol Med 24: 233246, 2009.

13. Matsuo S, Imai E, Horio M, et al: Revised equations for estimated GFR from serum creatinine in Japan. Am J Kidney Dis 53: 982-992, 2009.
14. Yamada Y, Fuku N, Tanaka M, et al: Identification of CELSR1 as a susceptibility gene for ischemic stroke in Japanese individuals by a genome-wide association study. Atherosclerosis: E-pub ahead of print, 2009.

15. Yoshida T, Kato K, Fujimaki T, et al: Association of genetic variants with chronic kidney disease in Japanese individuals. Clin J Am Soc Nephrol 4: 883-890, 2009.

16. Yoshida T, Kato K, Yokoi K, et al: Association of gene polymorphisms with chronic kidney disease in high-risk or low-risk subjects defined by conventional risk factors. Int J Mol Med 23: 785-792, 2009.

17. Itoh Y, Mizuki N, Shimada T, et al: High-throughput DNA typing of HLA-A, -B, -C and -DRB1 loci by a PCR-SSOP-Luminex method in the Japanese population. Immunogenetics 57: 717-729, 2005.

18. Suzuki S, Sano K and Tanihara H: Diversity of the cadherin family: evidence for eight new cadherins in nervous tissue. Cell Regul 2: 261-270, 1991.

19. Miotto E, Sabbioni S, Veronese A, et al: Frequent aberrant methylation of the $\mathrm{CDH} 4$ gene promoter in human colorectal and gastric cancer. Cancer Res 64: 8156-8159, 2004.

20. Obst-Pernberg K, Medina L and Redies C: Expression of $\mathrm{R}$-cadherin and $\mathrm{N}$-cadherin by cell groups and fiber tracts in the developing mouse forebrain: relation to the formation of functional circuits. Neuroscience 106: 505-533, 2001.

21. Cavodeassi F, Carreira-Barbosa F, Young RM, et al: Early stages of zebrafish eye formation require the coordinated activity of Wnt11, Fz5 and the Wnt/beta-catenin pathway. Neuron 47: 43-56, 2005.

22. Wasmeier C and Hutton JC: Molecular cloning of phogrin, a protein-tyrosine phosphatase homologue localized to insulin secretory granule membranes. J Biol Chem 271: 18161-18170, 1996.

23. Kawasaki E, Hutton JC and Eisenbarth GS: Molecular cloning and characterization of the human transmembrane protein tyrosine phosphatase homologue, phogrin, an autoantigen of type 1 diabetes. Biochem Biophys Res Commun 227: 440-447, 1996.

24. Orth SR: Smoking and the kidney. J Am Soc Nephrol 13: 1663-1672, 2002. 